\title{
Checklist of cockroaches (Blattodea) of Kerala, India
}

\author{
S. Prabakaran", M. Senraj" and Deepa Jaiswal \\ Freshwater Biology Regional Centre, Zoological Survey of India, Hyderabad 500048, \\ Telangana, India; "Southern Regional Centre, Zoological Survey of India, Chennai 600028, \\ Tamil Nadu, India. Email: prabakaranzsi@yahoo.co.in; sennraj@gmail.com
}

\begin{abstract}
Cockroach fauna comprises of 23 valid species under 14 genera. Information on the distribution, synonymy, type locality, type depository are provided. Thirteen species of blattids are added to the fauna of Kerala based on literature survey and study of specimens available in Western Ghat Field Research Centre, Zoological Survey of India, Kozhikode, Kerala. There are four species endemic to Kerala. () 2020 Association for Advancement of Entomology
\end{abstract}

KEYWORDS: Distribution, taxonomy, species of blattids

\section{INTRODUCTION}

The Order Blattodea, includes cockroaches and termites. It comprises of 4641 species of cockroaches under 492 genera in 8 families (Beccaloni, 2014) and 2929 species of termites belonging to 283 genera and 9 families (Krishna et al., 2013). In India, there are 181 species of cockroaches belonging to over 72 genera under 17 subfamilies in 6 families (Gupta and Chandra, 2019). The taxonomic work on Indian blattids still covers only common and easily available species. Despite having rich Indian blattid fauna especially in the forest ecosystem, taxonomy of many families of Blattodea in the country remains in a confused state, as studies on Indian blattids are scanty. From Kerala state, only 10 species of blattids were reported earlier by Gupta and Chandra (2019).

Initially Shelford (1910) described a species Haanina maindroni from Kerala, after that Mukherjee and Hazra (1991) recorded some species of cockroaches from Kerala i.e Haanina patinifera (Bolívar, 1897); Rhabdoblatta lineaticollis (Bolívar, 1897). Roth (1979a) recorded a few more species from Kerala namely Panesthia monstruosa Wood-Mason, 1876, Panesthia morosa Kirby, 1903 and Panesthiapara monstruosa Roth, 1979. Anisyutkin and Yushkova (2017) described three species from Kerala namely, Morphna indica, Rhabdoblattella alexeevi, Rhabdoblattella euptera. Study of specimens in the Western Ghat Field Research Centre, Zoological Survey of India, Kozhikode, Kerala (WGRC) and a detailed literature survey enhanced our knowledge of cockroach fauna of Kerala, adding 13 species now to the list. An updated checklist containing 23 species is enumorated.

\section{MATERIAL AND METHODS}

The present study is based on the literature on the cockroaches of Kerala (Shelford, 1910; Mukherjee 1989; Anisyutkin and Yoshkova, 2017; Mukherjee

* Author for correspondence

(C) 2020 Association for Advancement of Entomology 
and Hazra, 1991; Roth 1979, 1985; Grandcolas, 1993; Beccaloni, 2014) and by the study of specimens present in the National Zoological Collection at Western Ghat Field Research Centre Zoological Survey of India, (WGRC), Kozhikode, Kerala. The classification followed is based on Beccaloni and Eggleton (2013).

Abbreviations used in the text:

TL - Type locality

TD - Type depository. Acronyms for depositories

MNHN - National Museum of Natural History Paris.

CAS - California Academy of Sciences California

ZMUC - University of Copenhagen Zoological Museum

MHNG - The Natural History Museum of Geneva

MMUM - The Manchester Museum of the University of Manchester, Manchester, United Kingdom

BMNH - (British Museum of Natural History) Natural History Museum, in London.

LSUK - Linnaean Society, London, United Kingdom.

\section{RESULTS AND DISCUSSION}

Extensive literature and study of specimens helped in updating the cockroach fauna of Kerala. The updated list and systematic notes on additional 13 species are given (Table 1).

\section{Systematic Account}

Order BLATTODEA

\section{Superfamily: BLABEROIDEA Saussure, 1864}

Family: BLABERIDAE Saussure, 1864
Subfamily: EPILAMPRINAE Brunner von Wattenwyl, 1865

Tribe Morphnini McKittrick, 1964

Genus Haanina Hebard, 1929

1. Haanina maindroni (Shelford, 1910)

1910. Homalopteryx maindroni Shelford. Genera Insectorum 101: 7.

1967. Haanina maindroni Princis, Orthopterorum Catalogus (11): 644.

TL: India: Puducherry: Mahe (q)

TD: MNHN

Distribution: India: Puducherry: Mahe Remarks: Endemic to south India (Kerala).

2. Haanina patinifera (Bolívar, 1897)

1987. Homalopteryx patinifera Bolívar, Annls. Soc. Ent. Fr. 66: 295

1967. Haanina patinifera Princis, Orthopterorum Catalogus (11): 644.

TL: India: Tamil Nadu: Madurai ( $\$$ )

TD: MNHN

Distribution: India: Kerala; Tamil Nadu: Madurai; Lakshadweep Island.

Remarks: Endemic to south India.

Genus Morphna Shelford, 1910

3. Morphna decolyi (Bolivar, 1897)

1897. Molytria decolyi Bolívar. Annls. Soc. Ent. Fr. 66: 294.

1964. Stridoblatta decolyi McKittrick. Cornell Univ. Agric. Exp. Sta. Mem. 389: 44.

1910. Homolopteryx decolyi Shelford, Genera Insectorum 101: 7

1967. Haanina decolyi Princis, Orthopterorum Catalogus (11):644 
Table 1. Updated checklist of cockroaches of Kerala

\begin{tabular}{|c|c|c|c|}
\hline Sl. & Name of the species & References & Remarks \\
\hline & $\begin{array}{c}\text { Order BLATTODEA Brunner von } \\
\text { Wattenwyl. } 1882 \\
\text { Family BLABERIDAE Sassure, } 1864\end{array}$ & & \\
\hline 1. & Haanina maindroni (Shelford, 1910) & Shelford (1910) & Endemic to Kerala* \\
\hline 2. & Haanina patinifera (Bolívar, 1897) & Mukherjee and Hazra (1991) & Endemic to south India \\
\hline 3. & Morphna decolyi (Bolívar, 1897) & Anisyutkin (2014) & Endemic to south India \\
\hline 4. & Morphna indica Anisyutkin, 2017 & $\begin{array}{l}\text { Anisyutkin and } \\
\text { Yushkova (2017) }\end{array}$ & Endemic to Kerala* \\
\hline 5. & $\begin{array}{l}\text { Morphna plana } \\
\text { (Brunner von Wattenwyl, 1865) }\end{array}$ & $\begin{array}{l}\text { Identified specimens } \\
\text { from WGRC }\end{array}$ & Endemic to south Asia** \\
\hline 6. & $\begin{array}{l}\text { Rhabdoblatta lineaticollis } \\
\text { (Bolívar, 1897) }\end{array}$ & Mukherjee and Hazra, (1991) & Endemic to south India \\
\hline 7. & Stictolampra plicata (Navás, 1904) & Mukherjee (1989) & Endemic to India \\
\hline 8. & Thorax porcellana (Saussure, 1862) & Roth (1972) & - \\
\hline 9. & $\begin{array}{l}\text { Rhabdoblattella alexeevi } \\
\text { Anisyutkin, } 2017\end{array}$ & $\begin{array}{l}\text { Anisyutkin and } \\
\text { Yushkova (2017) }\end{array}$ & Endemic to Kerala \\
\hline 10. & $\begin{array}{l}\text { Rhabdoblattella euptera } \\
\text { Anisyutkin, } 2017\end{array}$ & $\begin{array}{l}\text { Anisyutkin and } \\
\text { Yushkova (2017) }\end{array}$ & Endemic to Kerala \\
\hline 11. & $\begin{array}{l}\text { Panesthia monstruosa } \\
\text { Wood-Mason, } 1876\end{array}$ & Roth (1979a) & Endemic to India* \\
\hline 12. & Panesthia morosa Kirby, 1903 & Roth (1979a) & Endemic to south India* \\
\hline 13. & Panesthia paramonstruosa Roth, 1979 & Roth (1979a) & Endemic to south India* \\
\hline 14. & Salganea erythronota Bolívar, 1897 & Roth $(1979 b)$ & Endemic to India* \\
\hline 15. & Salganea indica Princis, 1953 & $\begin{array}{l}\text { Identified specimens } \\
\text { from WGRC }\end{array}$ & Endemic to south India** \\
\hline 16. & $\begin{array}{l}\text { Pycnoscelus surinamensis } \\
\text { (Linnaeus, 1758) }\end{array}$ & Bolivar (1897) & Pest of Agriculture* \\
\hline \multirow[t]{2}{*}{17.} & Pycnoscelus indicus (Fabricius, 1775) & $\begin{array}{l}\text { Identified specimens } \\
\text { from WGRC }\end{array}$ & $\begin{array}{l}\text { Circumtropical- } \\
\text { Pest of Crops }{ }^{* *}\end{array}$ \\
\hline & $\begin{array}{c}\text { Family ECTOBIIDAE Brunner } \\
\text { von Wattenwyl, } 1865\end{array}$ & & \\
\hline 18. & $\begin{array}{c}\text { Blattella humbertiana (Saussure, 1863) } \\
\text { Family BLATTIDAE Latreille, } 1810\end{array}$ & Roth (1985) & - \\
\hline 19. & Hebardina concinna (Haan, 1842) & Hebard (1929) & - \\
\hline 20. & Neostylopyga rhombifolia (Stoll, 1813) & $\begin{array}{l}\text { Identified specimens } \\
\text { from WGRC }\end{array}$ & Circumtropical in Asia ${ }^{* *}$ \\
\hline 21. & $\begin{array}{l}\text { Neostylopyga ornata } \\
\text { (Brunner von Wattenwyl, 1865) }\end{array}$ & $\begin{array}{l}\text { Identified specimens } \\
\text { from WGRC }\end{array}$ & Endemic to south India** \\
\hline 22. & $\begin{array}{l}\text { Periplaneta americana } \\
\text { (Linnaeus, 1758) } \\
\text { Family CORYDIIDAE Saussure, } 1864\end{array}$ & $\begin{array}{l}\text { Identified specimens } \\
\text { from WGRC }\end{array}$ & Cosmopolitan species ${ }^{* *}$ \\
\hline 23. & Therea petiveriana (Linnaeus, 1758) & Grandcolas (1993) & Endemic to south India \\
\hline
\end{tabular}

* Species additional record to Kerala

** Species new record to south India 
1971. Morphna decolyi Princis, Orthopterorum Catalogus (14): 1156-57

TL: Tamil Nadu: Trichy (q)

TD: MNHN

Distribution: India: Kerala (current record); Tamil Nadu: Tiruchirappalli and Coimbatore.

Remarks: Endemic to South India.

4. Morphna indica Anisyutkin, 2017

2017. Morphna indica Anisyutkin in Anisyutkin and Yushkova, Zootaxa 4236 (1): 56.

Material Examined: $2 q$, India: Kerala, Chinnar Wild life sanctuary, Churulipetti, 04.iv.2012, coll. Sureshan, Reg.no. ZSI/WGRC/IR INV/ 14732.

TL: India: Kerala $(\hat{)})$

TD: MHNG

Distribution: India: Kerala: Cardamon Hills.

Remarks: Endemic to Kerala

5. Morphna plana (Branner von Wattenwyl,1865)

1865. Epilampra plana Brunner von Wattenwyl, Nouveau Système des Blattaires: 183.

1897. Homalopteryx biplagiata Bolívar. Annls. Soc. ent. Fr. 66: 296.

1967. Morphna plana Princis. Orthopterorum Catalogus (11): 648.

Material Examined: 1 $\hat{\jmath}$, India: Kerala, Idukki District, Mathikettan Sholai, 17.ix.2014;3 3̂, Idukki district, Eravikulam N.P, 23.ix.2014 coll. Emiliyamma Reg. nos. ZSI/WGRC/IR INV/14735, 14740; 1ठ, 1 , Idukki District, Erachippara, 23.ix.2014, coll. Emiliyamma, Reg.no.ZSI/WGRC/ IR INV/ 14824.

TL: Sri Lanka (ð)

TD: MMUM
Distribution: India: Kerala; Tamil Nadu: Tiruchirappalli. Elsewhere: Sri Lanka.

Remarks: Endemic to south Asia.

Genus Rhabdoblatta Kirby, 1903

6. Rhabdoblatta lineaticollis (Bolívar, 1897)

1897. Epilampra lineaticollis Bolívar. Annls Soc. Ent. Fr. 66: 298.

1904. Heterolampra lineaticollis Kirby, Syn. Cat. Orthop: 120.

1967. Rhabdoblatta lineaticollis Princis. Orthopterorum Catalogus (11): 672.

Material Examined: 19 , India: Kerala, Idukki district, Mannavan Shola, 06.iv.2012; coll. Sureshan; 19 , Idukki district, Mathikettan Sholai, 17.ix.2014, coll. Emiliyamma, Reg.nos. ZSI/WGRC/IR INV/ 14733, 14734

TL: India $(\hat{\jmath})$

TD: MNHN

Distribution: India: Kerala (Mukherjee and Hazra, 1991); Tamil Nadu: Trichirappali (Kirby, 1904)

Remarks: Endemic to south India.

Genus Stictolampra Hanitsch, 1930

7. Stictolampra plicata (Navás, 1904)

1904. Opisthoplatia plicata Navás. Bol. Soc. Aragon. Cienc. Nat. 3: 130.

1910. Rhicnoda plicata Shelford. Genera Insectorum 101: 9.

1967. Stictolampra plicata Princis, Orthopterorum Catalogus (11): 683.

TL: India: West Bangal: Kurseong $(\uparrow)$

TD: Unknown 
Distribution: India: Kerala, Arunachal Pradesh, Assam, Bihar, Chhattisgarh, Gujarat, Himachal Pradesh, Karnataka, Madhya Pradesh, Maharashtra, Meghalaya, Orissa, Punjab, Sikkim, Tripura, Tamil Nadu, Uttarakhand and West Bengal.

Elsewhere: Borneo; Java; Malayasia (Beccaloni, 2014)

Tribe THORACINI Rehn, 1951

Genus Thorax Saussure, 1862

8. Thorax porcellana (Saussure, 1862)

1862. Phoraspis (Thorax) porcellana Saussure, Rev. Mag. Zool. 2(14): 228.

1865. Paraphoraspis notata Brunner von Wattenwyl. Nouveau Système des Blattaires.: 164.

2014 Thorax porcellana Anisyutkin, Zootaxa 3847 (3): 321.

TL: India $(\hat{\jmath})$

TD: MHNG

Distribution: India: Kerala: Trivandrum, Cochin; Tamil Nadu: Coimbatore: Anamalai Hills, Nilgiris and Gudalur (Anisyutkin 2017); Goa; Karnataka: Mysore.

Elsewhere: Sri Lanka and Australia: Victoria (Beccaloni, 2014).

Genus Rhabdoblattella Anisyutkin, 1999

9. Rhabdoblattella alexeevi Anisyutkin, 2017

2017. Rhabdoblattella alexeevi Anisyutkin in Anisyutkin and Yushkova, Zootaxa 4236 (1): 48.

TL: India: Kerala (ठ)

TD: MHNG

Distribution: India: Kerala: Idukki district, Cardamon Hills, Mattupatti near Munnar.

Remarks: Endemic to Kerala.
10. Rhabdoblattella euptera Anisyutkin, 2017

2017. Rhabdoblattella euptera Anisyutkin in Anisyutkin and Yushkova, Zootaxa 4236 (1): 52.

TL: India: Kerala (ठ)

TD: MHNG

Distribution: India: Kerala: Idukki district, Cardamon Hills, Valara Falls.

Remarks: Endemic to Kerala.

Subfamily PANESTHIINAE

Genus Panesthia Serville, 1831

11. Panesthia monstruosa Wood-Mason, 1876

1876. Panesthia monstruosa Wood-Mason, Journ. Asiat. Soc., Bengal 15(2):189.

1897. Panesthia panteli Bolívar, Annls. Soc. Ent. Fr. 66: 307.

1979. Panesthia monstruosa Roth. Aust. J. Zool., Suppl. Ser., 74: 107.

Material Examined: 3 India: Kerala: Idukki Dist. Periyar Tiger Reserve, Eramangalar, 7.ix.2015, coll. Sureshan, Reg.no. ZSI/WGRC/IR INV/ 14822 .

TL: South India $(\hat{\jmath} q)$

TD: Unknown

Distribution: India: Kerala: Chalakudy, Cochin; Tamil Nadu: Coimbatore, Coonoor, Nilgiri, Anaikatti, Valparai, Thiruchirapalli, Madurai; West Bengal: Darjling: Kurseong; Sikkim (Roth, 1979).

Remarks: Endemic to India.

12. Panesthia morosa Kirby, 1903

1903. Panesthia morosa Kirby, Ann. Mag. nat. Hist. 7 (11): 412.

1979. Panesthia morosa Roth, Aust. J. Zool., Suppl. Ser., 74: 73. 
TL: India: Tamil Nadu ( $)$

TD: BMNH

Distribution: India: Kerala: Trivandrum; Tamil Nadu: Anaimalai Hills (Roth, 1979).

Remarks: Endemic to south India.

\section{Panesthia paramonstruosa Roth, 1979}

1979. Panesthia paramonstruosa Roth, Aust. J. Zool., Suppl. Ser., 74: 109.

Material Examined: 1 $\hat{\jmath}$, Eravikulam N.P, Mathikettan Sholai, Idukki District. 10.04.2012 coll. Sureshan, Reg.no. ZSI/WGRC/IR INV/ 14737; $1 \hat{\delta}$, Idukki District, Eravikulam N.P, Erachipara, 23.09.2014, coll. Emiliyamma, Reg.no. ZSI/ WGRC/IR INV/ 148372 2 , 2 Nymph Kambilipara Shola, Marayoor forest area, Idukki Dist. 20.05.2014. 1 J Kambilipara Shola, Marayoor forest area, Idukki Dist. 23.05.2014, coll. Sureshan, Reg.nos. ZSI/WGRC/IR INV/ 14820,14826

TL: India: Kerala: Munnar (亏)

TD: CAS

Distribution: India: Kerala: Munnar; Tamil Nadu: Anaimalai Hills, Anaikatti.

Remarks: Endemic to south India.

Genus Salganea Stål, 1877

14. Salganea erythronota Bolívar, 1897

1897. Salganea erythronota Bolívar, Annls. Soc. Ent. Fr. 66: 301.

1979. Salganea erythronota Roth. Australian Journal of Zoology Supp. 27(69): 94.

Material Examined: 29 , Mannavan Shola, 06.04.2012, coll. Sureshan, Reg.no. ZSI/WGRC/ IR INV/14736; 19 , Pambadum Shola N. P Idukki Dist. 26.5.2014, coll. Sureshan, Reg.no. ZSI/ WGRC/IR INV/ 14823; 2 exs. Pullaradi Shola, Mannavan Shola, Idukki Dist. 27.05.2014, coll. Sureshan, Reg.no. ZSI/WGRC/IR INV/ 1481919 ,
Chinna Anamudi, Idukki Dist, 20.09.2014, coll. Emiliyamma, Reg.no. ZSI/WGRC/IR INV/ 14821

TL: India: Tamil Nadu ( $($ )

\section{TD: MNHN}

Distribution: India: Kerala: Munnar; Tamil Nadu: Madurai: Shambaganaur, Trichy, Kodaikanal Hills, Palni Hills; Himalaya (Roth 1979a).

Remarks: Endemic to India.

15. Salganea indica Princis, 1953

1953. Salganea indica Princis, Opuscula Entomologica 18(1): 53.

1979. Salganea indica Roth, Australian Journal of Zoology Supp. 27(69): 63.

Material Examined: $1 \hat{\jmath}, 2 q, 9$ nymphs, India: Kerala, Kozhikode district, Malabar Wildlife sanctuary, Panikkar Kadavu, 19.03.2012, coll. Sureshan, Reg.no. ZSI/WGRC/IR INV/ 14739. 29,6 nymphs, Kattila Para, Kollam Dist. 21.01.2019, coll. Jafer Palot, Reg.no. ZSI/WGRC/ IR INV/ 14829

TL: India: Tamil Nadu (q)

TD: MNHM

Distribution: India: Kerala; Tamil Nadu: Anaimalai Hills; Chincona; Karnataka: Mysore (Roth 1979a).

Remarks: Endemic to South India.

Subfamily PERISPHAERINAE Brunner von Wattenwyl, 1865

Genus Pseudoglomeris Brunner von Wattenwyl, 1893

16. Pseudoglomeris sericea (Saussure, 1863)

1863. Perisphaeria sericea Saussure. Mém. Soc. Phys. Hist. Nat. Génève 17:138.

1863. Perisphaeria emortualis Saussure. Mém. Soc. Phys. Hist. Nat. Génève 17: 138. 


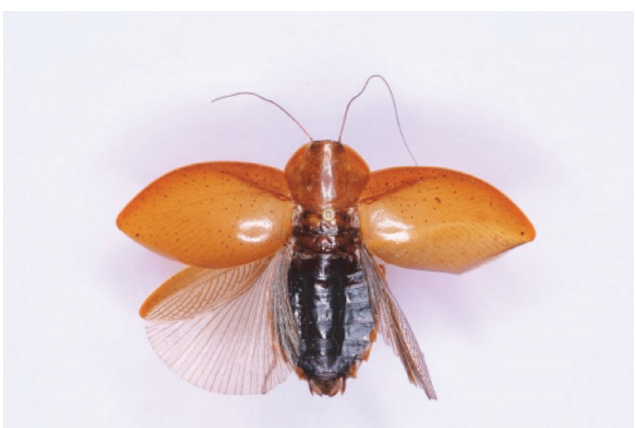

a) Dorsal view

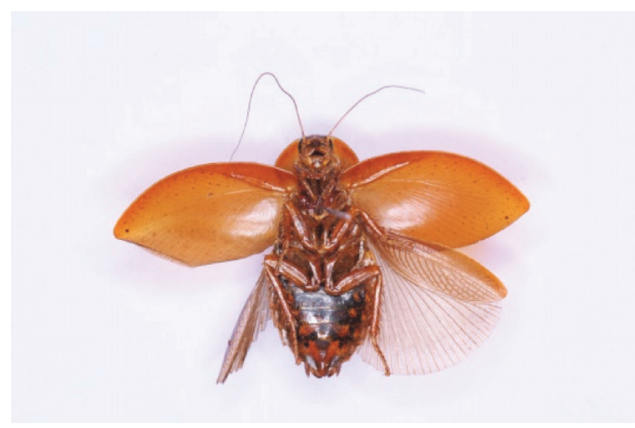

b) Ventral view

Fig.1. Thorax porcellana (Saussure, 1862)

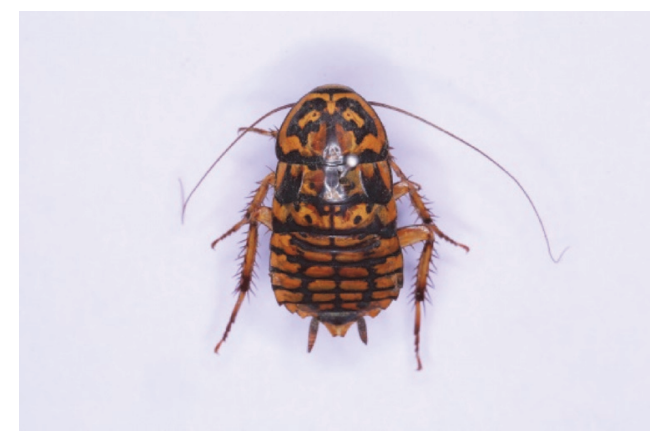

a) Dorsal view

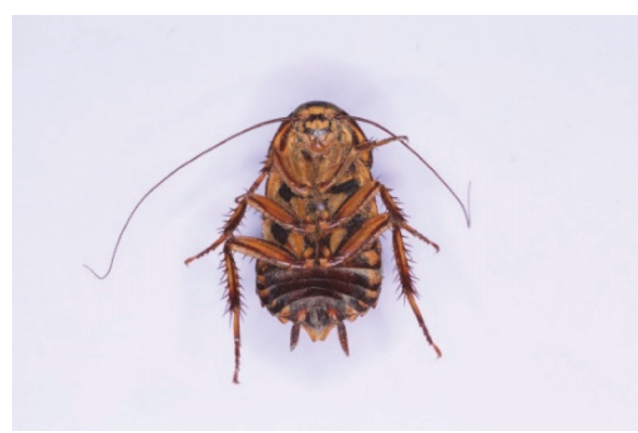

b) Ventral view

Fig.2. Neostylopyga rhombifolia (Stoll, 1813)

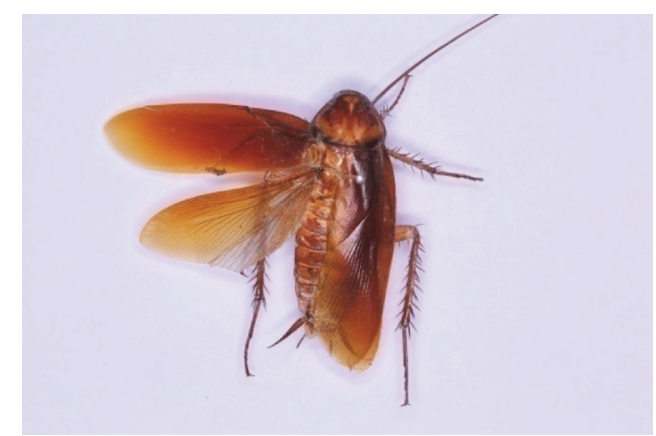

a) Dorsal view

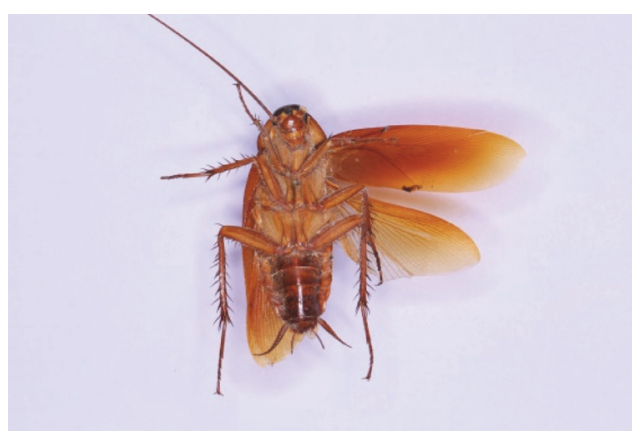

b) Ventral view

Fig. 3. Periplaneta americana (Linnaeus, 1758)

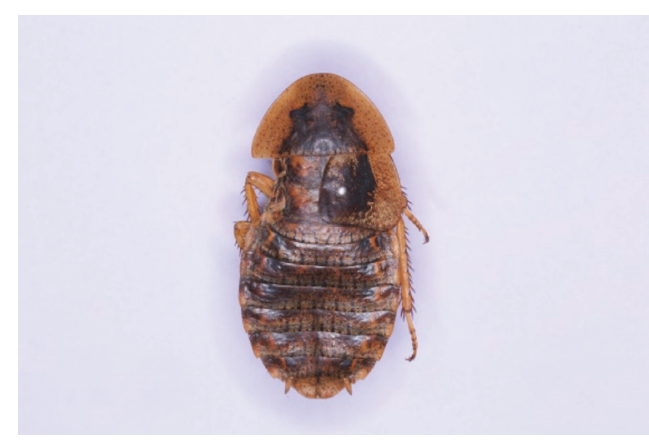

a) Dorsal view

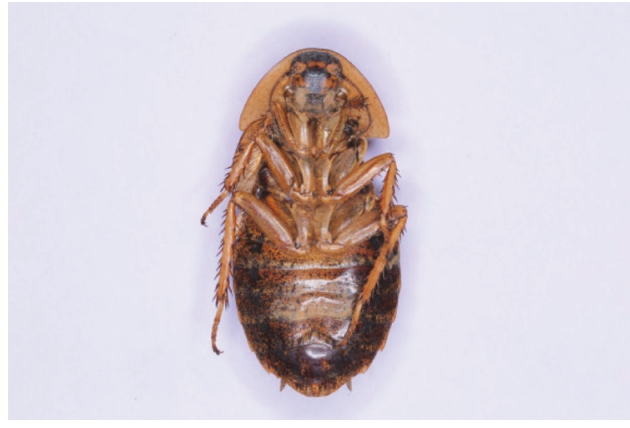

b) Ventral view

Fig. 4. Morphna indica Anisyutkin, 2017 


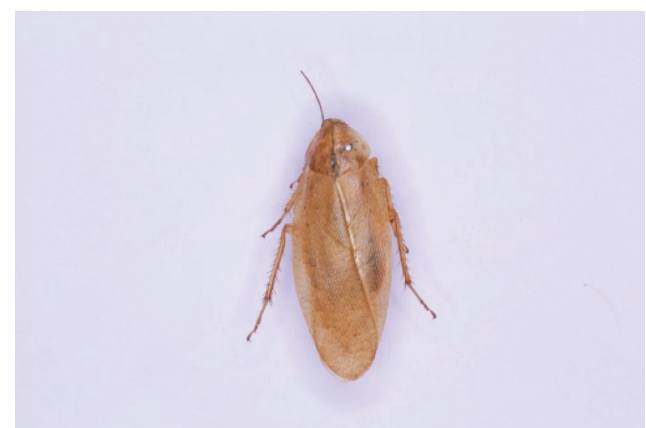

a) Dorsal view

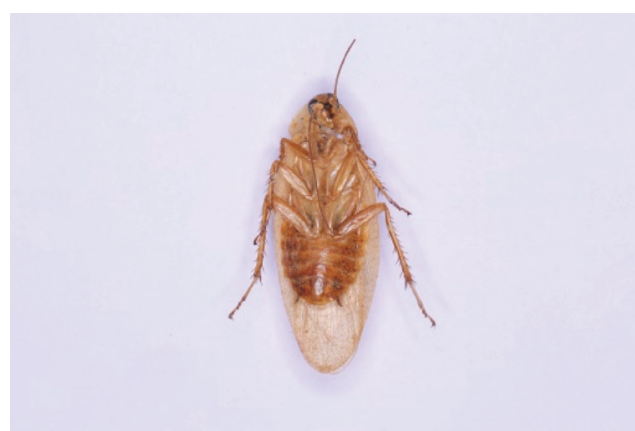

b) Ventral view

Fig. 5. Rhabdoblatta lineaticollis (Bolívar, 1897)

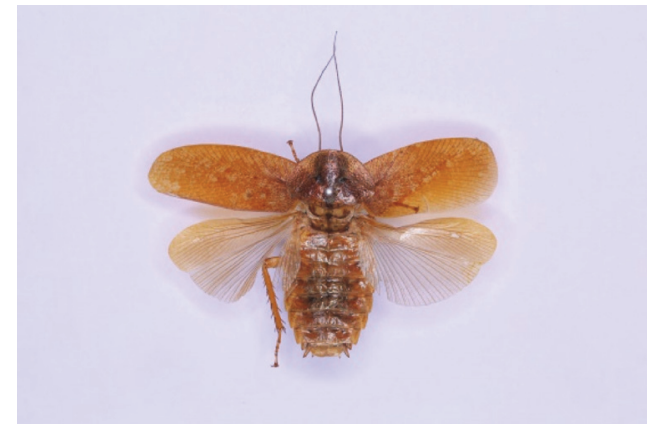

a) Dorsal view

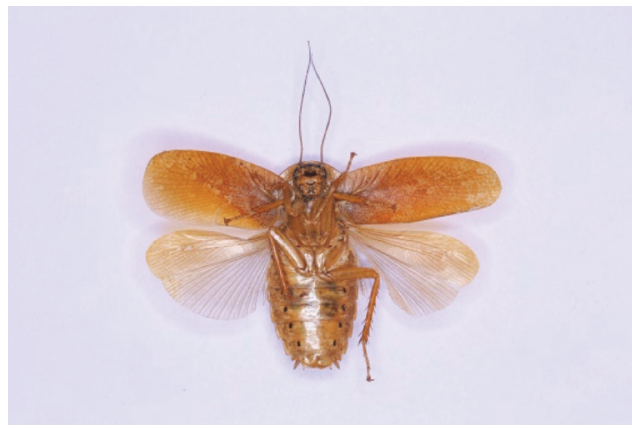

b) Ventral view

Fig.6. Morphna plana (Branner Von wattemay, 1865)

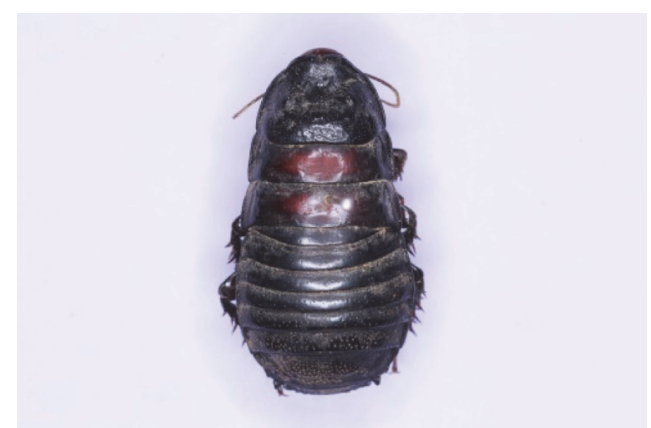

a) Dorsal view

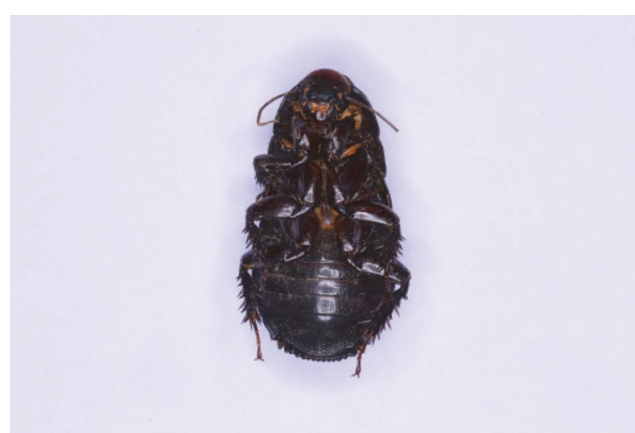

b) Ventral view

Fig.7. Salganea erythronota Bolívar, 1897

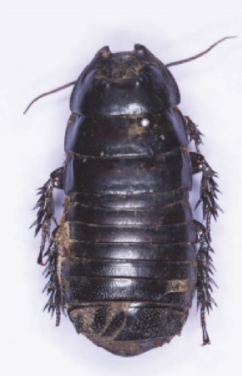

a) Dorsal view

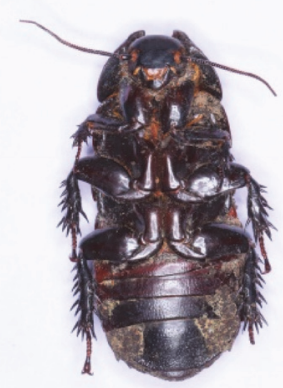

b) Ventral view

Fig. 8. Panesthia paramonstruosa Roth, 1979 


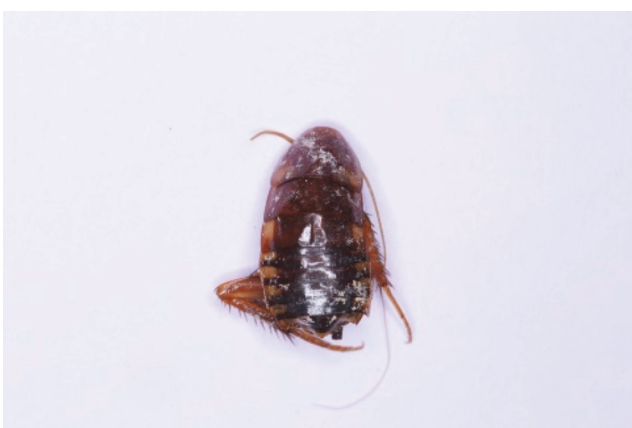

a) Dorsal view

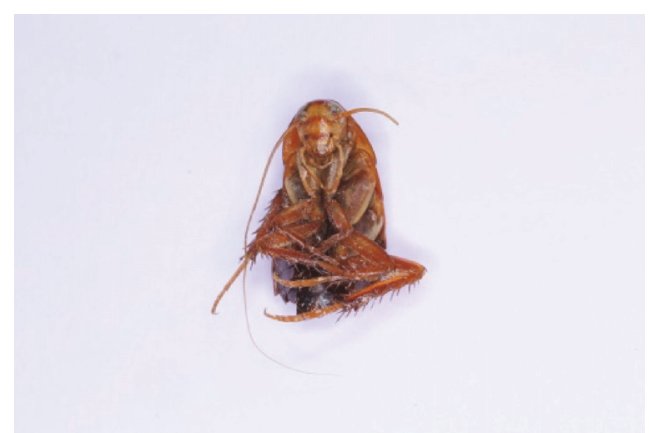

b) Ventral view

Fig. 9. Neostylopyga ornata (Brunner von Wattenwyl, 1865)

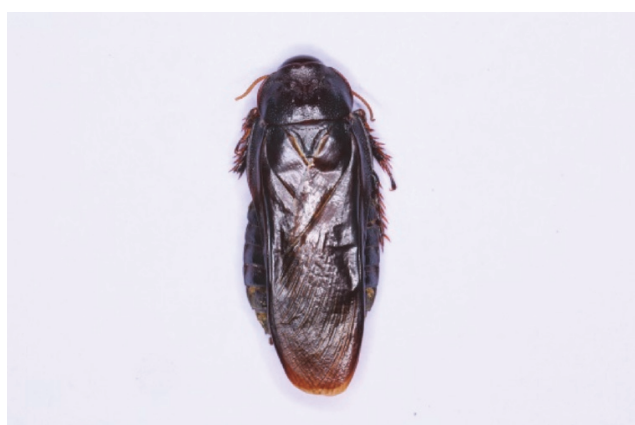

a) Dorsal view

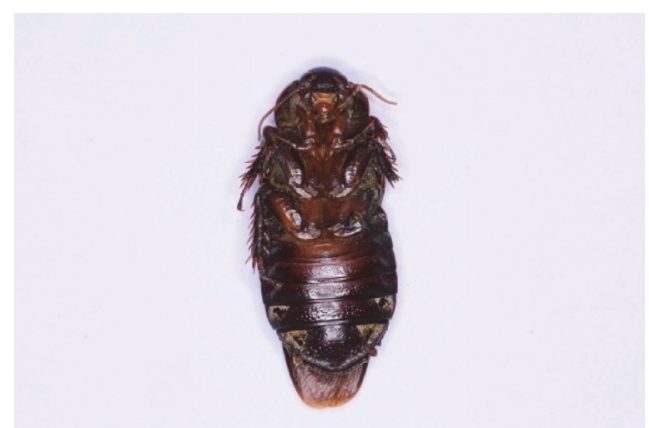

b) Ventral view

Fig. 10. Salganea indica Princis, 1953

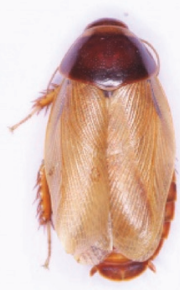

a) Dorsal view

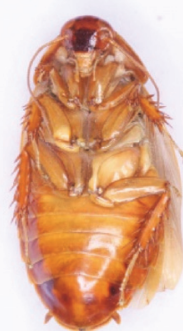

b) Ventral view

Fig. 11. Pycnoscelus indicus (Fabricius, 1775)

1964. Trichoblatta sericea Princis.

Orthopterorum Catalogus (6): 208.

2018. Pseudoglomeris sericea (Saussure, 1863) Xin-Ran Li et.al., Zootaxa 4410 (2): 259.

TL: India: Tamil Nadu (§)

TD: Unknown
Distribution: India: Kerala; Tamil Nadu; Puducherry; Andhra Pradesh; Bihar; Gujrat; Karnataka; Maharashtra; Orissa; Sikkim; West Bengal; Arunachal Pradesh; Meghalaya (Mukherjee, 1993).

Remarks: Endemic to India.

Subfamily PYCNOSCELINAE

Genus Pycnoscelus Scudder, 1862 
17. Pycnoscelus indicus (Fabricius, 1775)

1775. Blatta indica Fabricius, Syst. Ent.: 272.

1964. Pycnoscelus indicus Princis, Orthopterorum Catalogus (6): 270.

Material Examined: $1 q$, Pepparai dam, Trivandrum Dist, 13.10.2012, coll.Emiliyamma, Reg.no. ZSI/WGRC/IR INV/ 14827

TL: India

TD: ZMUC

Distribution in India: Kerala

Elsewhere: Sumatra, Thailand, Taiwan, Java, Philippines, USA, Burma, China \& Sri Lanka. Circumtropical (Beccaloni, 2014).

Remarks: Agricultural Pest.

\section{Family ECTOBIIDAE Brunner von} Wattenwyl, 1865

Subfamily BLATTELLINAE Karny, 1908

Genus: Blattella Caudell, 1903

18. Blattella humbertiana (Saussure, 1863)

1863. Polyzosteria humbertiana Saussure, Mém. Soc. Phys. hist. Nat. Génève 17: 131.

1865. Phyllodromia cognata Brunner von Wattenwyl. Nouveau Système des Blattaires.: 92.

1871. Blatta subreticulata Walker, Cata. Derm. Coll. Part V. Supp. Cata. Blat. 23.

1969. Blattella humbertiana Princis. Orthopterorum Catalogus (13): 842.

TL: Sri Lanka

TD: MHNG

Distribution: India: Kerala; Puducherry: Mahe (Roth,1985); Tamil Nadu; Bombay; Arunachal Pradesh; West Bengal; Andhra Pradesh; Arunachal Pradesh; Delhi; Karnataka; Maharashtra; Manipur; Punjab; Jharkhand; Uttarakhand; Delhi; Meghalaya; Orissa; Rajasthan and Tripura.
Elsewhere: Sri Lanka; Burma; China (Beccaloni, 2014).

Family Blattidae Latreille, 1810

Subfamily BLATTINAE Latreille, 1810

Genus Hebardina Bei-Bienko, 1938

19. Hebardina concinna (Haan, 1842)

1842. Blatta (Periplaneta) concinna Haan, Verhand. natuurl. (16) 6: 50.

1873. Periplaneta borrei Saussure. Mem. Soc. Geneve 23. 113.

1966. Hebardina concinna Princis, Orthopterorum Catalogus (8): 466.

1999. Hebardina concinna Roth, Oriental Insects, 33(1): 172.

TL: Indonesia: Java Island ( $\hat{\delta}+$ )

TD: Not Recorded

Distribution: India: Kerala (Mukherjee 1993); Tamil Nadu: Coimbatore: Anamalai Hills, Tiruchirappalli; Uttar Pradesh: Nainital district; Arunachal Pradesh; West Bengal: Howrah, Jalpaiguri and Kolkata.

Elsewhere: Burma; Malaysia (Malacca state); Indonesia (Sumatra); Indonesia (Java Island); Borneo Island (Beccaloni, 2014).

Remarks: Domiciliary pest

\section{Genus Neostylopyga Shelford, 1911}

20. Neostylopyga rhombifolia (Stoll, 1813) (Harlequin Cockroach)

1813. Blatta rhombifolia Stoll, Represent. exact. coloreed'apres nature d. Spectres: 5.

1966. Neostylopyga rhombifolia Princis. Orthopterorum Catalogus (8): 534.

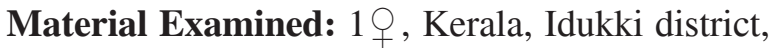
Chinnar Wildlife sanctuary, Vasyapara, coll. Sureshan, 05.v.2012 Reg.no. ZSI/WGRC/IR INV/ 14730 . 
TL: India: Bombay ( $($ )

\section{TD: BMNH}

Distribution: India: Kerala; Tamil Nadu: Chennai (Madras), Bombay. Meghalaya, Andaman and Nicobar Islands, Andhra Pradesh, Bihar, Madhya Pradesh, Orissa, Uttar Pradesh, and West Bengal; Himachal Pradesh, Circumtropical [Asian origin]. Elsewhere: Sri Lanka, Mauritius (East Africa), Borneo; Celebes; Java; Malayasia; Sarawak; Sumatra. America (Beccaloni 2014)

21. Neostylopyga ornata (Brunner von Wattenwyl, 1865)

1865. Periplaneta ornata Brunner von Wattenwyl, Nouveau Système des Blattaires.: 225.

1966. Neostylopyga ornata Princis. Orthopterorum Catalogus (11): 537.

Material Examined: 2 exs. Kerala, Idukki district, Chinnar W.L.S, Surulipatti, 22.05.2014, coll. Sureshan, Reg.no. ZSI/WGRC/IR INV/ 14738.

TL: India $(q)$

TD: Unknown

Distribution: India: Kerala; Tamil Nadu: Ramanathapuram: Kilakarai.

Remarks: Endemic to India: Tamil Nadu

Genus Periplaneta Burmeister, 1838

22. Periplaneta americana (Linnaeus, 1758)

1758. Blatta americana Linnaeus, Systema naturae 1, ed. 10, Holmiae: 424.

1966. Periplaneta americana (Linnaeus, 1758) Princis. Orthopterorum Catalogus (8): 405.

Material Examined: $1 \hat{\delta}$, Kerala, Kozhikode district, Kottooli wetlands, 9.12.2011 Coll. Sureshan, Reg.no. ZSI/WGRC/IR INV/ 14731

TL: America (ठ)

TD: LSUK
Distribution: India: Kerala; Tamil Nadu: Thiruchirapalli, Arunachal Pradesh: Papumpare district; West Bengal, Sikkim.

Elsewhere: America and Cosmopolitan [African origin].

Remarks: This is a cosmopolitan species and one of the most important domiciliary cockroach pests.

Superfamily Corydioidea Saussure, 1864

Family Corydiidae Saussure, 1864

Subfamily CORYDIINAE Saussure, 1864

Genus Therea Billberg, 1820

23. Therea petiveriana (Linnaeus, 1758)

1758. Cassida petiveriana Linnaeus, Syst. Nat: 364.

1767. Cassida septemguttata Linnaeus. Syst. Nat.: 577.

1963. Therea petiveriana Princis. Orthopterorum Catalogus (4): 84.

2008. Therea petriviana Fritzsche \& Zompro. Arthropoda 16(4):20.

TL: India ( $(9)$

TD: BMNH

Distribution: India: Kerala; Tamil Nadu: Coimbatore; Puducherry: Mahe.

Remarks: Endemic to India.

Based on a cursory survey of literature and by including the above mentioned 23 species, the checklist of cockroach fauna of Kerala is updated. Among these 10 species were listed earlier by Gupta and Chandra (2019) for Kerala, but their distribution within Kerala state was not mentioned. Study of specimens at WGRC has helped to bridge the gap. This checklist will be beneficial as a baseline data for future studies. Among the 23 species, 15 species are endemic to India in which eight species are endemic to south India and four species endemic to Kerala. 


\section{ACKNOWLEDGEMENTS}

We extend our sincere thanks to Dr. Kailash Chandra, Director, Zoological Survey of India, Kolkata for his continued encouragement and support for our research. We are also grateful to Dr. P.M. Sureshan, Scientist -E, Officer in Charges, Western Ghats Field Research Centre, Zoological survey of India, Kerala and Dr. K.A. Subramanian, Scientist-E, Officer in Charge, Southern Regional Centre, Zoological survey of India, Chennai for their support and encouragement to compile this work.

\section{REFERENCES}

Anisyutkin L.N. (2014) On cockroaches of the subfamily Epilamprinae (Dictyoptera: Blaberidae) from South India and Sri Lanka, with descriptions of new taxa. Zootaxa 3847 (3): 301-332.

Anisyutkin L.N. and Yushkova O.V. (2017) New data on cockroaches of the subfamily Epilamprinae (Dictyoptera: Blaberidae) from India and Sri Lanka, with descriptions of new species and the genital complex of Aptera fusca (Thunberg, 1784). Zootaxa 4236 (1): 41-64.

Beccaloni G.W. and Eggleton P. (2013) Order Blattodea. Zootaxo 3703(1): 46-48. DOI: 10.11646 / Zootax. 3703.1.10

Beccaloni G.W. (2014) Cockroach Species File Online. Version 5.0/5.0. World Wide Web electronic publication. <http://Cockroach.SpeciesFile.org> [accessed 01 December 2018].

Bolívar I. (1897) Les Orthopteres de St-Joseph's College a Trichinoploy (Sud de I'Inde). Annals of Soceity of Engomology, France 66: 282-316.

Grandcolas P. (1993) Le genre Therea Billberg, 1820: position phylogenetique, nouvellesespkces, repartition, valence ecologique (Dictyoptera, Blattaria, Polyphaginae). Canadian Journal of Zoology 71: 1816-1822.

Gupta S.K and Chandra K. (2019) An annotated checklist of cockroaches (Blattodea) from India. Zootaxa
4614(3): 461-497. DOI: 10.11646/zootaxa.4614.3.3.

Hebard M. (1929) Studies in Malayan Blattidae (Orthoptera). Proceedings of the Academy of Natural Sciences of Philadelphia 81: 1-109.

Kirby W.F. (1904) A synonymic catalogue of Orthoptera. British Museum of Natural History 1: 1-501.

Krishna K., Grimaldi D.A., Krishna V. and Engel M.S. (2013) Treatise on the Isoptera of the world. Bulletin of the American Museum of Natural History, 377(1-7): 1-2704.

Mukherjee K.P. (1989) Insecta: Dictyoptera: Blattariae. In: State Fauna Series. Vol. 1. Fauna of Orissa. Part 2. Zoological Survey of India, Kolkata. pp. 171-179.

Mukherjee K.P. and Hazra A.K. (1991) Insecta: Dictyoptera. In: State Fauna Series. Vol. 2 Fauna of Lakshadweep. Zoological Survey of India, Kolkata. pp. 263-264.

Roth L.M. (1972) The Male Genitalia of Blattaria IX. Blaberidae. Gyna spp. (Perisphaeriinae) Phoraspis, Thorax, and Phlebonotus (Epilamprinae). Transactions of the American Entomological Society 98(2): 185-217.

Roth L.M. (1979) A Taxonomic Revision of the Panesthiinae of the World III. The Genera Panesthia Serville and Miopanesthia Serville (Dictyoptera: Blattaria: Blaberidae). Australian Journal of Zoology Suppliment series 74: 1-276.

Roth L.M. (1979a) A Taxonomic Revision of the Panesthiinae of the World II. The Genera Salganea Stal Microdina Kirby and Caeparia Stal (Dictyoptera: Blattaria: Blaberidae). Australian Journal of Zoology Suppliment series 69: 1-201.

Roth L.M. (1985) A taxonomic revision of the genus Blattella Caudell (Dicyopter, Blattaria: Blattellidae). Entomologica Scandinavica Supplement 22:1-221.

Shelford R. (1910) Orthoptera. Fam. Blattidae. Subfam. Epilamprinae. In: Wytsman, P. (Ed.), Genera Insectorum 101: 1-21. 